\title{
Audrey GILLES
}

\section{La poésie du phallus dans Le Condamné à mort de Jean Genet}

En 1942, Jean Cocteau écrit dans son journal à propos du Condamné à mort: "Pour moi ses poèmes sont le seul grand événement de l'époque. En outre, protégés par l'érotisme (impubliables), ils ne peuvent que se lire en cachette, que se glisser de la main à la main. » (1989, p. 269) Ces mots reflètent parfaitement la dimension subversive de la poésie de Genet et, en particulier, du Condamné à mort, première œuvre de l'auteur, écrite en prison. En dépit de sa facture classique - le poème est composé en grande partie de quatrains en alexandrins au mètre rigoureux —, le texte de Genet joue avec la norme et ses écarts, avec la transgression, en mettant en scène deux thèmes sulfureux : la sublimation du criminel prisonnier et les amours homosexuelles.

La place qu'accorde le poète au corps est révélatrice de cette volonté paradoxale de s'inscrire à la fois dans une tradition poétique - la lyrique amoureuse - et contre une norme sociale. Le corps masculin est en effet un champ où le fantasme et l'imaginaire se déploient. Le corps est morcelé pour permettre une pluralité du chant poétique et une focalisation érotique. Cette focalisation est portée en particulier par l'image du phallus. Comme l'a noté Hélène Cixous, il y a une véritable «pénilisation générale»(1981-1982, p. 60) dans le texte de Genet. Si cette présence du sexe masculin, en particulier dans un contexte homosexuel et souvent argotique, n'est pas une nouveauté en poésie - nous songeons par exemple aux Ballades en jargon de Villon ou encore au recueil Hombres de Verlaine - , elle n'est pas, chez Genet, seulement ludique. Le phallus renvoie dans Le Condamné à mort au domaine du sacré. En érigeant, dans son poème, une partie «honteuse » du corps en quasidivinité et en maittre du chant, Genet renouvelle la lyrique amoureuse tout en affichant la magnificence de l'érotisme homosexuel. La provoca- 
Audrey GILlES, «La poésie du phallus dans Le Condamné à mort de Jean Genet », @nalyses, automne 2007

tion et la subversion poétiques sont alors des moyens de questionner la norme, qu'elle soit esthétique, sociale ou poétique.

En mettant en avant un aspect particulier de l'esthétique de Genet et de l'imaginaire du Condamné à mort - le pouvoir érotique et subversif du sexe masculin - il s'agira d'observer comment le phallus prend, dans ce poème, la place de maître du chant et comment, à travers ses mises en scène, ses rapports à la norme et sa sacralisation, il est le sujet principal de la création poétique en même temps que son origine.

\section{Mises en scène du phallus}

Le sexe masculin apparait dans le poème comme une obsession. Le nommer, pour le poète, revient à l'incarner dans un poème parcouru par l'angoisse de la mort et par l'urgence du désir. Qu'il apparaisse en tant que simple partie du corps permettant l'accès au plaisir ou comme métaphore de la structure du poème, le phallus est, à l'image du « torse tatoué », ce « poteau sacré » qu'il faut adorer.

Le sexe masculin est envisagé sous des formes multiples. Douze mots différents sont ainsi employés pour le désigner dans le poème: « verge » (strophe 8), « chibre » (strophe 15), « queue » (strophes 17, 26 et 29), «braguette » (strophe 20), "membre» (strophe 28), "paf» (strophe 29), "bite» (strophes 29 et 47), « sexe » (strophe 30), "bâton» (strophes 30 et 47), «vit» (strophe 54), « pine» (strophe 61). Trois d'entre eux seulement appartiennent au langage courant « verge », « membre » et « sexe » — alors que tous les autres sont argotiques ou imagés. La diversité des termes, en particulier en argot, permet d'inscrire le poème dans un milieu interlope, celui des bagnards. Dire le sexe est alors aussi bien un code qu'une provocation pour le lecteur étranger à ce milieu. Genet tient son lecteur à l'extérieur de son poème par la crudité et le sujet de son propos : le sexe est de l'ordre de l'intimité de la cellule pénitentiaire et ne s'affiche d'abord au lecteur-voyeur que comme une provocation. L'environnement lexical de ces occurrences met en évidence deux points essentiels : l'indépendance du phallus par rapport au reste du corps, sa personnification en même temps qu'une immobilité absolue. 
Audrey GILlES, «La poésie du phallus dans Le Condamné à mort de Jean Genet », @nalyses, automne 2007

Ainsi, de nombreux adjectifs qualifiant ordinairement l'être humain sont attribués au sexe, tels que « adorable », « insolent », " émouvant »; des subordonnées relatives viennent également indiquer une action réalisée par le sexe : "qui monte », "qui [...] te frappe ». La seule occurrence qui rend une immobilité — «mon vit de marbre noir» — est lourde de sens : le phallus est ici assimilé à une statue, à la pierre d'un autel, voire d'une pierre tombale. Il est ici une divinité de pierre à adorer.

Cette problématique de la cellule qui fait naitre une frontière entre le poète, son sujet et le lecteur est particulièrement présente dans la mise en scène du sexe. Celui-ci apparaît comme le personnage d'une pièce de théâtre érotique dont le lieu de représentation serait la cellule close du prisonnier. Le lecteur n'est alors plus seulement voyeur; il est spectateur d'une pièce dont le sujet et le seul acteur sont un phallus. En effet, lorsque Genet met en scène ses personnages, il se focalise sur leur désir et leur plaisir. En cela, leur sexe devient le symbole, la réduction de la tension érotique. L'acte sexuel, signifié par des queues, des bouches et des culs, est dépouillé, et le lecteur-spectateur est attiré par la représentation comme il en est rejeté. La strophe 29 révèle parfaitement cette obsession phallique :

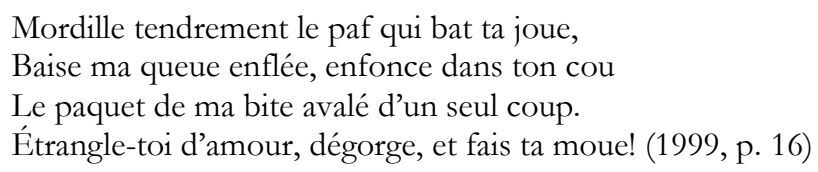

La violence de la vision, rendue par la familiarité des mots et les impératifs, installe une relation ambiguë avec le lecteur. À la fois repoussé, choqué par la vulgarité première - la désignation du sexe masculin revient sous trois formes argotiques différentes en l'espace d'une seule strophe - et hypnotisé par l'expression saisissante du désir et d'une relation homosexuelle, le lecteur, œil du voyeur collé à la serrure de la cellule ${ }^{1}$,

\footnotetext{
${ }^{1}$ Nous renvoyons ici au personnage du gardien dans le film de Genet, Un Chant d'amour. Dans ce court-métrage muet et en noir et blanc, réalisé en 1950, Genet met principalement en scène trois personnages, liés les uns aux autres uniquement par le fantasme : deux prisonniers (un jeune et un plus âgé) séparés par le mur de leur cellule et un gardien. Celui-ci, au début du film, passe devant chaque cellule et
} 
Audrey Gilles, «La poésie du phallus dans Le Condamné à mort de Jean Genet », @nalyses, automne 2007

est cependant condamné à rester à l'extérieur de celle-ci. La strophe est à la fois obscène et pudique; la cellule-strophe est toujours voilée d'un mystère : on ne peut la pénétrer. Le lecteur reste étranger au drame intime, à la passion des deux amants prisonniers, comme l'écrit Albert Dichy: «[...] Genet parle toujours de l'autre côté d'un mur, toujours séparé de celui à qui il s'adresse; comme si cette séparation était l'espace même de sa parole.» (1987, p. 41) Cet espace entre l'intériorité et l'extériorité est l'espace poétique, celui dans lequel peut se réaliser la tension entre dedans et dehors, happant et rejetant le lecteur. La cellule devient un espace scénique, le lecteur un spectateur et le poète est à la fois dramaturge et thaumaturge : il ordonne la cellule-strophe, l'organise, crée et matérialise l'érotisme à travers la structure poétique. La récurrence du sexe masculin dans la strophe 29 est ainsi un moyen de concrétiser l'acte sexuel par l'écriture : "paf », «queue » et «bite » sont à la fois employés pour leur crudité et pour mettre en avant le phallus au centre de la strophe-cellule. C'est vers lui que tendent tous les désirs et tous les regards, si bien qu'il s'en trouve à la fois incarné et désincarné : les gestes se démultiplient « mordille », «baise », " avalé » — et seule la dimension obsessionnelle du sexe demeure, "dressé seul au milieu» de la cellule comme du vers: «paf», «queue » et «bite» sont en effet placés soit en milieu de vers, à l'hémistiche, soit au centre de l'hémistiche.

Cette question de l'agencement du vers met par ailleurs en évidence le traitement métaphorique du phallus. En effet, celui-ci devient l'image même du vers poétique. Dans «L'Étrange mot d'... », Genet évoque ainsi son rapport à la langue française et, en particulier, aux mots :

[...] cette langue comme les autres permet que se chevauchent les mots comme des bêtes en chaleur et ce qui sort de notre bouche c'est une partouze de mots qui s'accouplent [...].

observe par la lucarne des portes le spectacle érotique offert par les prisonniers : l'un se masturbe en se lavant, un autre en dansant, un autre encore en rêvant... La tension érotique passe ici seulement par le regard, et les courts instants saisis par le gardien au cœur des cellules provoqueront en lui un désir de pénétrer à l'intérieur de ces cellules, en particulier dans celle du prisonnier âgé. Sa position d'observateur est alors double : il fait à la fois son travail de gardien en s'assurant que tout se déroule bien dans la prison et se trouve également dans une position de voyeur, fantasmant sur un monde qui lui est refusé, l'univers érotique des prisonniers. 
Audrey GILlES, «La poésie du phallus dans Le Condamné à mort de Jean Genet », @nalyses, automne 2007

\begin{abstract}
Si quelqu'un espère qu'au moyen d'une telle prolifération — ou luxuriance de monstres il pourra soigner un discours cohérent, il se trompe : au mieux il accouple des troupeaux larvaires et sournois pareils aux processions des chenilles processionnaires, qui échangeront leur foutre pour accoucher d'une portée carnavalesque sans portée réelle, sans importance, issue du grec, du saxon, du levantin, du bédouin, du latin, du gaélique, d'un chinois égaré, de trois mongols vagabonds qui parlent pour ne rien dire mais pour, en s'accouplant, révéler une orgie verbale dont le sens se perd non dans la nuit des temps mais dans l'infini des mutations tendres ou brutales. (2002, p. 887)
\end{abstract}

Les mots ont un sexe, une dimension érotique frénétique qui empêche tout ordre, toute raison du texte. Cependant, c'est dans leur agencement que peut apparaître un sens. Dans Le Condamné à mort, le vers témoigne de cette luxuriance, de cette dimension sexuelle de la langue. Il se fait tour à tour «tendre ou brutal». Le vers-phallus est mouvant, ambigu; c'est lui qui donne à la cellule-strophe sa vitalité, mimant les vibrations charnelles d'un corps sexué et empli de désir qui se fait poétique.

Certaines différences entre la première édition du poème, en 1942, et les suivantes sont révélatrices de cette organisation poétique obsessionnelle autour du sexe masculin. Ainsi, dans un vers, certains mots — souvent des adjectifs possessifs — ont été modifiés pour permettre un resserrement sur le narrateur; on trouve, par exemple, dans la première édition : «Suce son membre comme on suce un glaçon». Le «son » disparaîtra au profit de «mon» dans les éditions suivantes, la focalisation change et le corps, par cette transformation, prend une matérialité plus saisissante. Le changement est le même dans un vers de la strophe suivante. Alors que l'on trouve "Baise sa tête enflée » dans l'édition de 1942, les éditions suivantes ont retenu «Baise ma queue enflée ». La modification ici est double : la première va dans le sens de notre exemple précédent avec le changement de «sa» en «ma» et la seconde modification est d'ordre lexical puisque c'est «queue » et non « tête » que l'on peut lire. Le vers s'est encore érotisé, poussé vers un aspect beaucoup plus charnel et cru que dans la première version. En resserrant le vers sur le narrateur et sur l'acte sexuel, Genet lui donne une proximité corporelle : on passe d'un «membre» sinon indifférencié, du moins lointain, à un sexe «immédiat». L'intimité est plus palpable, plus dérangeante aussi, car plus proche. Ce n'est plus seulement la focalisation qui est rétrécie, mais le vers tout 
Audrey GILlES, «La poésie du phallus dans Le Condamné à mort de Jean Genet », @nalyses, automne 2007

entier: chaque vers, par ce rapprochement du narrateur, semble acquérir une certaine indépendance. Chaque vers se dresse ainsi dans le poème comme autant de sexes en érection, d'où la régularité des alexandrins et le peu de rejets ou de contre-rejets, qui forceraient la destruction de l'unité du vers.

Dans ses mises en mots, en scène et en vers, le phallus s'impose par la crudité du langage et des visions proposées par le poète. C'est la subversion qui apparait en premier lieu au lecteur, jusque dans l'utilisation d'un mètre régulier pour signifier le désir. Genet propose ainsi, grâce à l'image récurrente du sexe masculin, une réflexion poétique sur la norme.

\section{Le phallus et la norme}

Le Condamné à mort, contrairement aux textes de Villon et de Verlaine et contre une tradition poétique paillarde — les poèmes de Maupassant par exemple - , est avant tout un chant d'amour plutôt qu'une approche ludique de la représentation sexuelle. Ainsi, il se nourrit aussi bien de la norme, la lyrique amoureuse, que de ce qui peut en constituer un écart.

L'image du phallus est dans le poème en concurrence avec la représentation florale. Ce motif est récurent dans l'œuvre de Genet, ne seraitce que dans les titres de ses romans, par exemple Miracle de la rose ou encore Notre-Dame-des-Fleurs. En tant que topos de la poésie amoureuse héritée du pétrarquisme, la métaphore florale est le témoignage, dans Le Condamné à mort, de cette tradition poétique. Neuf fleurs différentes sont ainsi évoquées : «roses» (strophes 4 et 37), « rosiers» (strophe 5), «fleurs de violettes» (strophe 10), « œillets » (strophe 14), « jasmins » (strophe 14), « lilas » (strophe 17), «fleurs » (strophes 36, 43 et 45), «fleurir» (strophe 36), "résédas » (strophe 43). Leur utilisation dans le corps du poème est souvent métaphorique. Prendre la fleur comme élément de comparaison revient, pour le poète, à s'inscrire dans une tradition culturelle à la fois littéraire et religieuse. La fleur devient un ornement, un artifice du désir, alors que le sexe est une résurgence primitive, une pulsion instinctive, ce qui explique la violence des mots employés pour l'évoquer. Les deux aspects du désir ne sont pourtant pas totalement opposés dans le texte: Genet les entre- 
Audrey GILlES, «La poésie du phallus dans Le Condamné à mort de Jean Genet », @nalyses, automne 2007

mêle pour créer une image particulière de l'érotisme: "Enfant d'honneur si beau couronné de lilas / Penche-toi sur mon lit, laisse ma queue qui monte»(1999, p. 14). Ici, le sexe est cérémonial; la couronne de fleurs renforce la métaphore religieuse. L'aspect sacré de l'érotisme, dans ce premier vers, est associé à une dimension profane dans le deuxième vers : le sexe caressant la joue de l'amant est à la fois une adoration et une profanation. Par ailleurs, ce parallélisme montre l'émergence d'un nouveau topos, propre à l'univers de Genet. Le sexe remplace peu à peu les fleurs dans son imaginaire amoureux et érotique. Le phallus est ainsi à la lyrique amoureuse homosexuelle ce qu'était la fleur dans la lyrique amoureuse pétrarquiste. Il y a une véritable contamination du floral par le sexuel, et ce, notamment dans les structures poétiques où la fleur avait l'avantage : la comparaison et la métaphore. Si, chez Ronsard ou du Bellay par exemple, la femme pouvait être comparée à une rose, l'amant chez Genet est assimilé à un sexe : le «petit mec» est ainsi «plus émouvant et pur qu'une émouvante bite ». La norme florale est détournée, mais ce que crée Genet est une norme qui lui est propre, une norme interne à son œuvre, celle de l'omniprésence du sexe comme élément d'analogie.

La présence phallique, loin de cloisonner la lecture dans l'éloge d'une virilité stéréotypée, amène à une transformation du masculin, non en féminin, mais en un genre troublé, androgyne. Le phallus est ce qui permet ce glissement et c'est ce à quoi s'est intéressée Elizabeth Stevens dans son article "Disseminating Phallic Masculinity: Seminal Fluidity in Genet's Fiction » (2004) ${ }^{2}$. Se posant contre un consensus de

${ }^{2}$ «Rather than seeing the phallus as the sign of a masculinity that is impenetrably self-contained, always erect and powerful, Genet represents the phallus as that which opens masculinity to transformations, flowing across its apparently sealed borders. [...] In emphasising this metamorphic aspect of phallic masculinity - the way it trembles, quivers, responds to touch, grows erect, discharges Genet's representations of the penis do not reproduce the traditional interpretation of the phallus as a symbol of stable and self-contained male corporeality, but rather draw attention to its inherent volatility. " Je traduis : «Plutôt que de voir dans le phallus le signe d'une masculinité, comme s'il était mystérieusement autonome, toujours puissant et en érection, Genet représente le phallus comme ce qui ouvre la masculinité à la transformation, débordant de ses frontières apparemment closes. En soulignant l'aspect protéiforme de la masculinité phallique 
Audrey GiLles, «La poésie du phallus dans Le Condamné à mort de Jean Genet», @nalyses, automne 2007

la critique pensant que l'érotisation du sexe masculin chez Genet ne fait que renforcer une vision conventionnelle et hétérosexuelle de la virilité, Elizabeth Stephens montre au contraire que les descriptions du phallus introduisent un processus de déstabilisation de cette image traditionnelle de l'homme. En effet, le processus rituel de l'acte sexuel mène à une métamorphose du pénis :

Il bondit sur tes yeux; il enfile ton âme

Penche un peu la tête et le vois se dresser.

L'apercevant si noble et si propre au baiser

Tu t’inclines très bas en lui disant : « Madame! » (p. 16)

Le «il», dans cette strophe, renvoie au sexe évoqué dans la strophe précédente. Le phallus, ici, n'est ni rigide ni statique, il est en mouvement, il mène seul le désir et l'acte sexuel : «il bondit », « il enfile », il se dresse et n'a pas seulement une puissance charnelle : il est associé à une image obsédante — «il bondit sur tes yeux» — qui tire l'amant vers un ailleurs spirituel : «il enfile ton âme». Le sexe est à la fois marqué par sa crudité et par son immatérialité : incarné par des «queue », «bite» ou "paf» dans tout le poème et par des vers qui miment ses convulsions par une rapidité de rythme et un ton haché, il se désincarne dans sa dimension poétique et fantasmatique pour finalement devenir, à la fin de cette strophe, une femme. Par la révérence et la dénomination du phallus par «Madame », le sexe garde de sa noblesse, de sa puissance fascinatrice, mais son caractère masculin se trouble : il n'est donc pas adoré pour sa stabilité, son immuabilité virile, mais pour sa capacité à se transformer. La métamorphose phallique bouleverse alors le corps et l'identité même de l'amant et des amants : à leur tour, ils subissent le trouble, se désincarnent physiquement pour être saisis dans un érotisme universel, au-delà des corps et de la réalité. La chair n'est pas annihilée : elle est renforcée par l'aspect sacré que lui donne la métamorphose. La norme du genre, masculin et féminin, comme celle de la préférence sexuelle, homosexualité ou hé-

- la façon dont le phallus frémit, tremble, réagit au toucher, entre en érection, décharge -, les représentations du pénis par Genet ne reproduisent pas l'interprétation traditionnelle du phallus comme symbole d'une réalité corporelle stable et autonome du mâle, mais attirent plutôt l'attention vers la mutabilité qui lui est inhérente. » (2004, p. 88) 
térosexualité, sont ainsi troublées. L'omniprésence du sexe masculin n'est donc pas dictée par une volonté phallocrate. Au contraire, elle permet une désorganisation sexuelle et, par là même, sociale. Ce qui importe alors dans la représentation du sexe n'est pas tant son genre que sa capacité poétique à faire surgir la beauté et l'émotion.

L'image du phallus dans Le Condamné à mort permet de questionner la norme aussi bien dans la tradition poétique que dans ses répercussions sociales. Ce que Genet dessine à travers l'emploi récurrent du sexe masculin, c'est une poésie paradoxale qui semble se dégager de la norme par la subversion, tout en ayant besoin d'elle pour créer une ritualité et une dimension sacrée, essentielle à l'imaginaire du poète.

\section{Le sacre du phallus}

Le poème de Genet semble s'inscrire dans la tradition antique de la procession phallique : l'exhibition poétique du membre, son association avec les fleurs, font de lui la divinité suprême de l'imaginaire du poète. Le phallus est au centre de rites, à la fois religieux et sexuels, qui mènent à la découverte d'un ailleurs.

Le passage du poème le plus signifiant à propos de la sacralisation du phallus au sein d'un rituel est sans doute celui de la cigarette. Organisé autour de la figure d'un jeune prisonnier, «le mino blond», ce rituel, qui a pour objet premier la distribution de la fumée d'une cigarette, a un pouvoir érotique très fort. Le passage s'ouvre sur la préparation du rituel à travers une parade de l'attente :

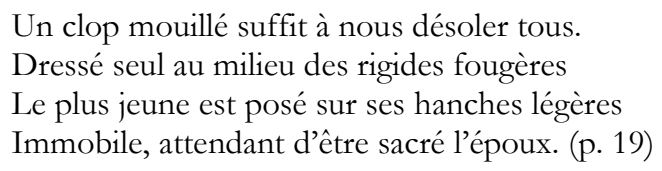

Cette strophe donne une première vision de l'élu qui va devenir divinité. Son corps organise la scène et la strophe : son immobilité, relayée par ce qui l'entoure — «rigides fougères » — suspend la temporalité, inscrit le corps dans une éternité. Cette immobilité est, d'autre part, une première annonce de la compréhension symbolique du rite : c'est 
Audrey GILlES, «La poésie du phallus dans Le Condamné à mort de Jean Genet », @nalyses, automne 2007

une initiation sexuelle que Genet masque sous le rite de la cigarette. Ainsi, de nombreux aspects phalliques sont présents à travers les adjectifs «dressé », « rigides », « immobile ». Le sexe, en érection, se tient prêt. Cette initiation est présentée comme une noce (" époux ») sacrée, homosexuelle et mystique, où les genres sexuels se troublent.

La suite du passage oppose la fragilité féminine ainsi que la jeunesse de l'élu à la force et à l'âge des bagnards : "les vieux assassins » comme «le bandit le plus dur» s'inclinent devant «le gamin frêle». Genet renverse ainsi le principe initiatique de l'homosexualité dans la Grèce antique, où les plus âgés "éduquaient» les jeunes garçons. Le «mino blond» se change en un nouvel Éros, érigé en divinité des amours homosexuelles et qui transmet le désir et le plaisir par la fellation.

Graves, silencieux, à tour de rôle enfant,

Vont prendre sur ta bouche une goutte embaumée,

Une goutte, pas deux, de la ronde fumée

Que leur coule ta langue. O frangin triomphant (p. 20)

La solennité des bagnards semble dérisoire en regard de l'objet du rite : la fumée d'une cigarette. Mais ce qui est en jeu, nous l'avons vu, est tout autre. Tout comme les bacchanales qui étaient non seulement des cultes religieux, mais aussi des moments de transe érotique, où le désir se déchaînait, fumer, dans le texte, revient à accomplir un désir érotique. La parade sexuelle est dissimulée derrière une parade prosaïque, profane, mais dans laquelle le fantasme est palpable sous les mots, ambigus : «bouche », "goutte », «coule », «langue » peuvent s'appli-quer à une description de la fellation. Seul le terme de « fumée » brise l'explicitation, bien que cette substance soit immédiatement associée au sperme ${ }^{3}$. Cette image de la fellation est d'ailleurs entièrement explicitée dans la strophe qui suit ce rituel: «Viens couler dans ma bouche un peu de sperme lourd » (p. 20). Le phallus, dans cette scène, n’est jamais évoqué. La cigarette devient son substitut et seules les

\footnotetext{
${ }^{3}$ L'image de la fumée comme symbole du sperme que deux prisonniers se font couler bouche à bouche renvoie à l'une des scènes clés du film de Jean Genet, Un Chant d'amour, où deux prisonniers se font passer la fumée d'une cigarette au travers du mur qui les sépare grâce à une paille enfoncée dans un trou. Cette image est une récurrence fantasmatique de l'univers de Genet.
} 
Audrey GILlES, «La poésie du phallus dans Le Condamné à mort de Jean Genet », @nalyses, automne 2007

parties du corps qui lui sont associées dans la fellation, la bouche et la langue, sont évoquées. Rite d'initiation, cette parade de la cigarette est un rituel érotique dans lequel l'objet principal, le sexe masculin, est détourné et sublimé.

Ce qu'il faut cependant retenir de ce rituel, c'est la communion qui va naittre et l'explicitation qui va se faire dans la suite du poème. En effet, les strophes qui suivent lèvent toute ambiguité : c'est bien une scène de fellation qui est décrite par Genet.

Élève-toi dans l'air de la lune, ô ma gosse.

Viens couler dans ma bouche un peu de sperme lourd

Qui roule de ta gorge à mes dents, mon Amour,

Pour féconder enfin nos adorables noces. (p. 20)

Cette strophe n'oppose pas le charnel au spirituel, mais montre l'accomplissement de l'un par l'autre. Genet mêle dans l'image de la fellation un aspect sacré et une réalité crue; ou plutôt, il encadre la matérialité de l'acte — «bouche», «sperme lourd», « gorge», «dents» - par une élévation mystique. Les rimes embrassées illustrent parfaitement cette structure. Le premier vers, par l'ambiguïté de ses mots, démultiplie les interprétations : qui s'élève? La réponse la plus évidente est celle donnée par "gosse », puis par l'impératif du deuxième vers, «Viens »: le poète semble alors s'adresser à l'amant. Le plaisir qui les lie, la jouissance qui fait s'élever l'amant dans la nuit est une communion; les «noces » sont alors profanes; l'union est érotique et se mêle à une image de la communion chrétienne. L'échange des fluides corporels mime celui des hosties. Tout comme ces derniers sont les incarnations du corps du Christ, le sperme est une matérialisation du désir qui habite les amants.

La fécondation qui est à l'œuvre est celle du poète par ses fantasmes. L'acte sexuel est communion car il est processus de création. Les « noces » sont cet espace mental où se rejoignent les deux amants, où le chant d'amour peut se faire célébration d'une jouissance à la puissance créatrice. 
Audrey GILlES, «La poésie du phallus dans Le Condamné à mort de Jean Genet », @nalyses, automne 2007

Il y a dans Le Condamné à mort une jouissance de l'écriture, un plaisir des mots, une dimension charnelle qui rapproche la création poétique de l'acte sexuel. L'amant est une figure du poète, les lieux du plaisir sexuels sont ceux de la naissance du chant: "Tristesse dans ma bouche!» (p. 13) La plainte du prisonnier est ici à prendre dans deux sens explicités par le dernier vers de la strophe : "O sur ma voix coupée adieu chibre insolent! » (p. 13) La bouche est à la fois le lieu de la fellation et celui de la voix, du chant. L'adieu au sexe de l'amant est aussi un adieu à la puissance créatrice du prisonnier-poète. Le vocabulaire employé pour parler du chant et du sexe est souvent le même, ce qui permet de semer la confusion et d'ouvrir les significations, comme dans la strophe 17 : «Penche-toi sur mon lit, laisse ma queue qui monte / Frapper ta joue dorée. Écoute, il te raconte ». Il est frappant de constater que, dans ce contexte de création, le verbe «monte» peut aussi bien être assimilé au sexe qu'au chant qui commence à s'élever, si bien que la fin du troisième vers poursuit cette ambiguiité. Si le pronom «il » renvoie à l'amant, évoqué dans le vers suivant, ce pourrait également être la «queue » — masculinisée alors en chibre, sexe, paf — qui « raconte ». Le phallus apparaît comme l'élément indispensable à la création; c'est par lui que s'opère l'élaboration poétique. Ainsi, Genet fait de ce qui est normativement honteux et dénué de beauté, le phallus, le principe même de sa poésie: «Il naîtra de son corps d'étonnantes splendeurs, / Du foutre parfumé de sa queue adorable » (p. 15). Ces vers reposent sur un principe alchimique que mettent en évidence le verbe naître ainsi que les compléments de nom introduits par la préposition de, qui indique une provenance. La «queue» peut être prise pour une métaphore du poème: ainsi, les «étonnantes splendeurs » et le «foutre » sont des images de la beauté qui jaillit du texte poétique travaillé. Chanter le sexe et l'ériger en source poétique permet, la mort approchant, de vaincre celle-ci.

Chez Genet, le phallus n'est plus seulement envisagé sous un aspect ludique où l'argot est à la fois une provocation et un moyen de se protéger de l'extérieur. Chanter le sexe masculin, c'est pour Genet donner une place à une beauté autre — comme ont pu le faire les Romantiques par exemple en exploitant la figure du criminel —, une beauté qui tient tout entière dans une réduction stéréotypée de la masculinité. Chanter 
Audrey GILlES, «La poésie du phallus dans Le Condamné à mort de Jean Genet », @nalyses, automne 2007

le phallus, ce n'est pas porter aux nues la virilité, mais c'est exprimer avec tendresse et violence le désir homosexuel. Le sexe, dans la facture classique du poème, entre en concurrence avec des topoï poétiques amoureux tels que les fleurs. L'être aimé n'est plus une femme; il faut, pour signifier ce changement, un symbole fort, rôle que prend le phallus, qui devient dans Le Condamné à mort une métaphore aussi bien du désir que de la création poétique. La séparation des genres s'estompe et, plus qu'une réflexion sur l'homo-érotisme, c'est une réflexion sur un érotisme universel que propose Genet. Le phallus, par son pouvoir poétique, est ce par quoi advient une norme mouvante et insaisissable. Objet de toutes les convoitises, sujet de tous les chants d'amour, le sexe, que l'obsession rituelle du poète tente de retenir par les mots, ne cesse de s'échapper, laissant le désir seul comme moteur d'écriture.

\section{Bibliographie}

CIXOUS, Hélène. 1981-1982, «Entretien sur Genet », Masques, revue des homosexualités, $\mathrm{n}^{\circ} 12$, hiver, p. 59-63.

COCTEAU, Jean. 1989, Journal 1942-1945, Paris, Gallimard.

DICHY, Albert. 1987, «Jean Genet : la prison imaginaire », Magazine littéraire, $\mathrm{n}^{\circ} 247$, novembre, p. 39-41.

GENET. Jean. 1999, Le Condamné à mort et autres poèmes suivi de Le Funambule, Paris, Gallimard, coll. «Poésie/Gallimard ».

—. 2002 [1967], « L'Étrange mot d'... », dans Théâtre Complet, Paris, Gallimard, coll. «Bibliothèque de la Pléiade ».

STEPHENS, Elizabeth. 2004, « Disseminating Phallic Masculinity: Seminal Fluidity in Genet's Fiction », Paragraph, No. 27, July, p. 85-97. 